\title{
Metastasis to the proximal ureter from prostatic adenocarcinoma: A rare metastatic pattern
}

\author{
Tao Zhang, MD; Qi Wang, MM; Jie Min, MD; Dexin Yu, MD; Dongdong Xie, MD; Yi Wang, MD; \\ Demao Ding, MD; Lei Chen, MD; Ci Zou, MD; Zhiqiang Zhang, MM; Daming Wang, MM
}

Department of Urology, The Second Hospital of Anhui Medical University, China

Cite as: Can Urol Assoc J 2014;8(11-12):e859-61. http://dx.doi.org/10.5489/cuaj.2169 Published online November 24, 2014.

\section{Abstract}

Prostate cancer is one of the most common male malignancies, but it rarely metastasizes to the proximal ureter. We report a case of a 76-year-old man who presented with flank pain and lower urinary tract symptoms. Abdominal computed tomography scan revealed multiple filling defects at the middle of the left ureter, enlarged retroperitoneal lymph nodes, and probable psoas invasion. The patient underwent nephroureterectomy with excision of a cuff of bladder, and was found to have an adhesion between the middle part of left ureter and psoas intraoperatively. The pathological examination displayed positive immunohistochemical staining with prostate-specific antigen and prostate acid phostate, supporting the diagnosis of metastatic ureteral tumour from prostate cancer. In this case, periureteral soft tissue and ureteral muscular layer were infiltrated by metastatic tumour, whereas the mucosa was spared. The periureteral lymphatic pathway played an important role in the metastatic procedure of prostate cancer to the proximal ureter.

\section{Introduction}

Prostate cancer is the second most common cancer in men. ${ }^{1}$ Its incidence has been decreasing in many developed countries, yet increasing rapidly in some Asian countries. ${ }^{2}$ The most common metastatic targets for prostate cancer include lymph nodes, bone, lung, and liver; however, metastasis to the proximal ureter is unusual. We present a case of ureteral tumour caused by metastasis of prostate cancer, and review the literature to discuss the possible pathway of this metastatic procedure.

\section{Case report}

In February 2012, a 76-year-old man presented with intermittent pain of the left flank lasting 1 month. He also had a 2-year history of mild obstructive urinary symptoms. He denied hematuria and body weight loss, and his medical and family history was unremarkable. Laboratory workup demonstrated no microscopic hematuria by urinary analysis, and a normal level of alkaline phosphates. Ultrasonographic examination demonstrated only mild hydronephrosis, with a dilated upper ureter on the left side. Cause of the dilatation was a stricture of the lower part of the ureter. To identify the nature of the stricture, we conducted a computed tomography $(\mathrm{CT})$ scan of the patient's abdomen. It revealed multiple filling defects at the middle part of left ureter with mild hydroureteronephrosis proximal to the site of stricture, enlarged retroperitoneal lymph nodes, and probable psoas invasion (Fig. 1). It was diagnosed as primary urothelial cell carcinoma of left ureter, although cytology detected some squamous, but not malignant, cells.

Because of the symptomatic hydronephrosis and suspected urothelial cell carcinoma of the left ureter, nephroureterectomy with a bladder cuff excision was done. Intraoperatively, he had an adhesion between the middle part of the left ureter and stiff left psoas, which we speculated was primary urothelial cell carcinoma of the left ureter invading the nearby psoas due to the rarity of the metastatic ureteral tumour. The pathological examination of the ureteral mass, however, revealed the adventitia and the muscular wall of the left ureter invaded by malignant cells. He was then diagnosed with prostate adenocarcinoma, as a result of the positive immunohistochemical staining for prostate-specific antigen (PSA) and prostate acid phostate (PAP) (Fig. 2).

Subsequently, the following tests were done: serum PSA value which was $>100 \mathrm{ng} / \mathrm{mL}$; a digital rectal examination (DRE) which revealed a hard rocky prostate on the bilateral side; and a transrectal ultrasound (TRUS)-guided prostate biopsy. Pathology was significant for adenocarcinoma of the prostate (Gleason score $3+4=7$ ). Concurrent whole body bone scan was also completed which provided the evidence of diffuse skeletal metastasis, including skull, left scapula, right sacroiliac joint, and ischial tuberosity.

To delay the progression of primary prostate cancer and multiple skeletal metastases, we performed medical castra- 


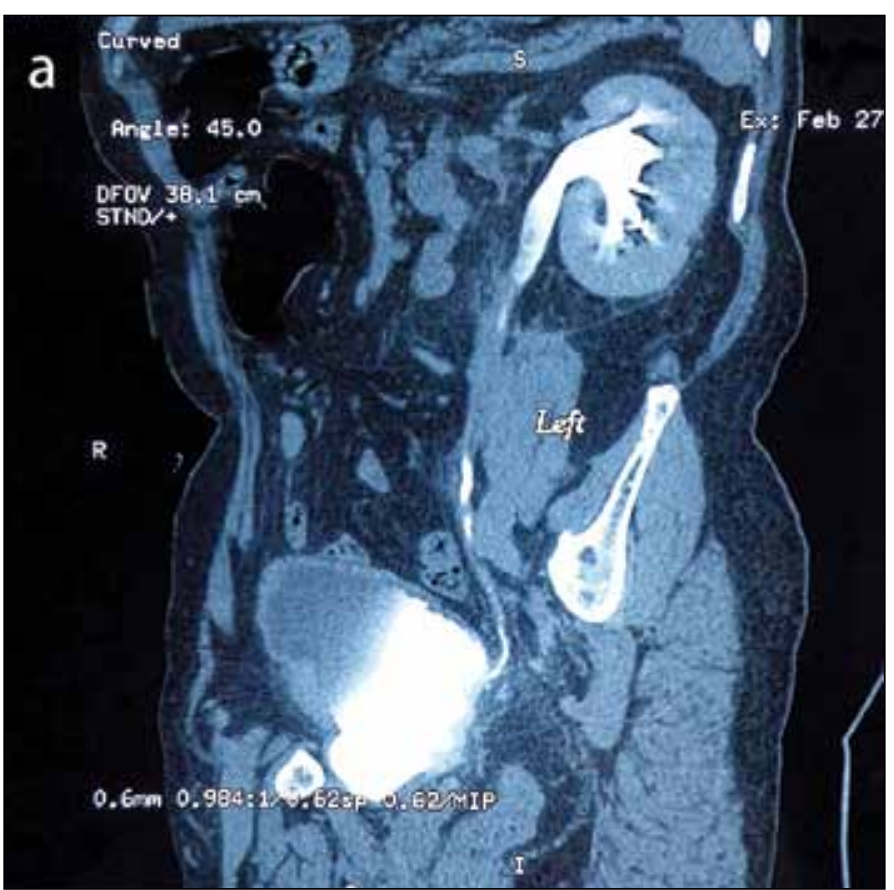

Fig. 1a. A computed tomography scan showing multiple filling defects at the middle of left ureter, and probable left psoas invasion.

tion. The patient was later discharged and received clinical follow-up. His PSA level decreased to $18.14 \mathrm{ng} / \mathrm{mL} 1$ month later, and then to $1.62 \mathrm{ng} / \mathrm{mL} 3$ months later.

\section{Discussion}

Prostate cancer is the second most frequently diagnosed cancer and the sixth leading cause of cancer death among men worldwide. ${ }^{1}$ It commonly spreads to regional lymph nodes via lymphatic vessels, direct invasion to pelvic organs or sys-

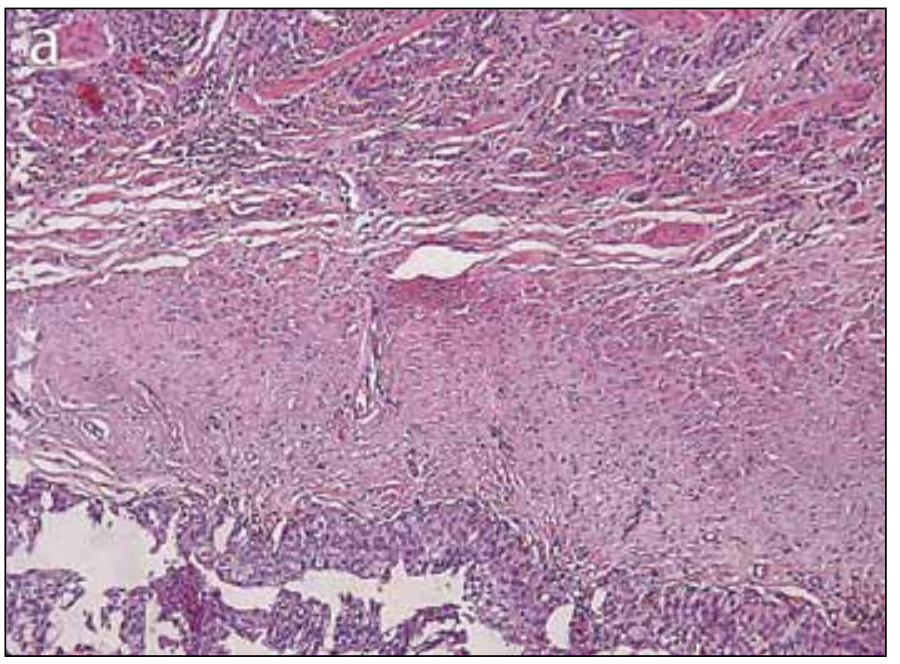

Fig. 2a. Hematoxylin-eosin appearance of metastatic ureteral tumour, the adventitia and muscular layer of ureter invading by malignant cells, but ureteral mucosa was spared $(\times 100)$.

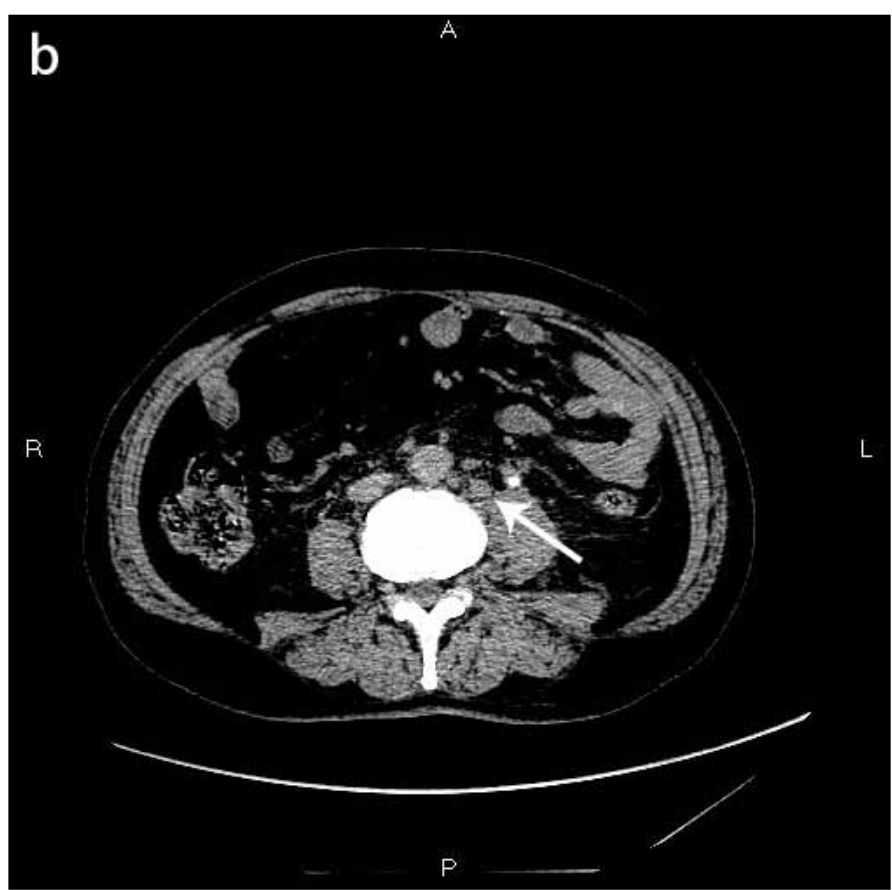

Fig. 1b. A computed tomography scan showing enlarged retroperitoneal lymph nodes (arrow).

temically to the axial skeleton. ${ }^{3}$ In an autopsy study, Disibio and colleagues ${ }^{4}$ found that the 5 most common metastatic targets for prostate cancer included regional lymph nodes $(26.2 \%)$, bone $(19.7 \%)$, distant lymph nodes $(18.4 \%)$, lung $(12.8 \%)$, and liver $(7.8 \%)$.

Upper urinary tract urothelial tumours involving the ureter are relatively uncommon compared with renal pelvis and true metastatic ureteral tumours from other primary cancers are especially rare. ${ }^{5}$ Stow $^{6}$ reported the first case of metastatic ureteral tumour from a lymphosarcoma in 1909. The

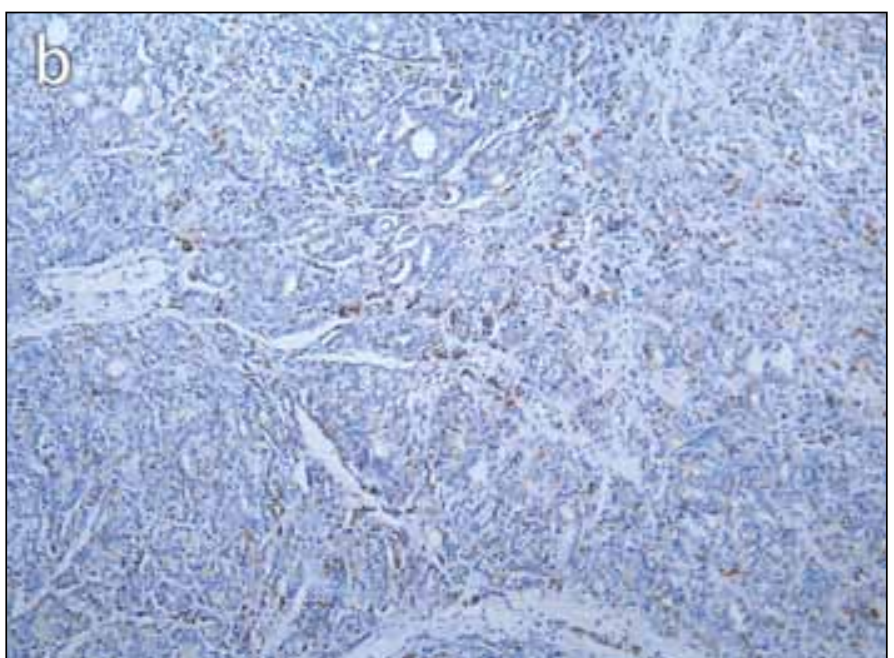

Fig. $\mathbf{2 b}$. Immunohistochemical staining of ureteral tumour positive for prostatespecific antigen $(\times 100)$. 


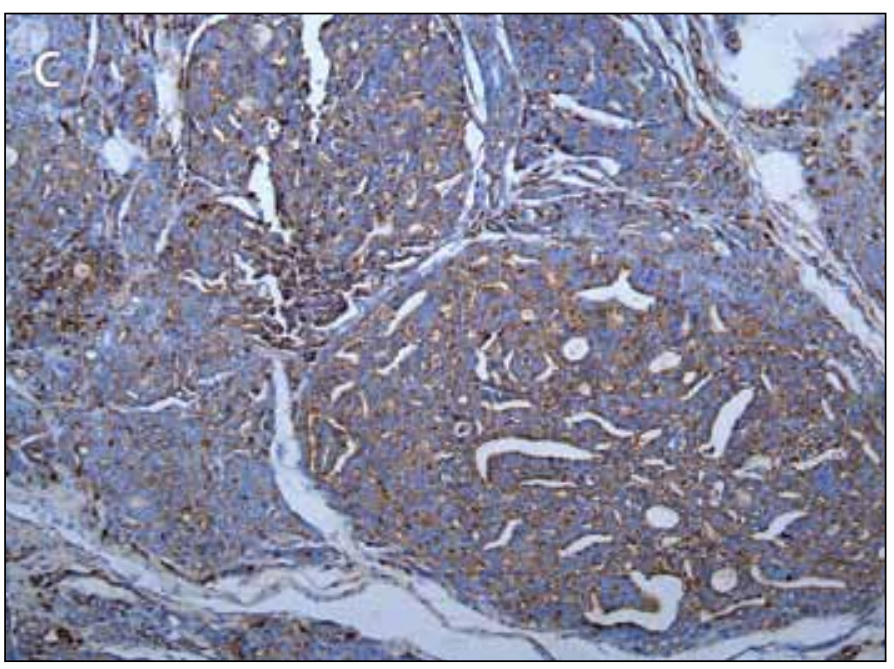

Fig. 2c. Immunohistochemical staining of ureteral tumour positive for prostate acid phostate $(\times 200)$.

definition for metastatic tumour to the ureter is well-documented by Hulse and $\mathrm{O}^{\prime} \mathrm{Neill}^{7}$ cases involving the ureter by direct extension or contiguity must be excluded. Cohen and colleagues ${ }^{8}$ reviewed the relevant literature and found 31 cases of true ureteral metastases; of these, the most common site of the primary lesion included the breast, colon, lymphoma, and lung - none from the prostate.

Ureteral obstruction caused by prostate cancer frequently results from invasion of the ureter by direct extension of the tumour around the intravesical ureter. Therefore, it is usually bilateral and mostly located at the ureterovesical junction. ${ }^{9}$ As noted previously, it is rate that metastatic tumour from the prostate to the proximal ureter happens without direct extension. The rarity is presumed to be due to the lymphatic circulation of the ureter, which is segmental and drains diagonally or transverse; there is no continuous longitudinal lymphatic network draining directly from the prostatic area. ${ }^{7}$ Ureteric stricture can also be caused by prostate cancer through other patterns. Chalasani and colleagues reported a case of prostate cancer with continuous invasion of the entire length of the ureter up to the renal pelvis, and no enlarged lymph nodes near the ureter - in this case the predisposing factors were unknown. ${ }^{10}$ In our case, the abdominal CT scan showed multiple enlarged lymph nodes in the retroperitoneal space around the left ureter, and the patient had an adhesion between the middle part of the left ureter and a stiff left psoas at surgery, yet the distal ureter was normal. Pathological examination of the ureteral mass revealed the adventitia and the muscular wall of the ureter invaded by malignant cells; however, the ureteral mucosa was unaffected. It is reasonable to believe that prostatic malignant cells were disseminated to the retroperitoneal lymph nodes near the proximal ureter through the periureteral lymphatic pathway; enlarged lymph nodes then compressed the ureteral lumen and ultimately extended to the ureter per continuitatem. They did not go through the lymphatic network in the ureteral wall or continued on the invasion pathway.

Prostate cancer should be considered in the differential diagnosis of elderly men presenting with ureteral tumour, especially in those with enlarged periureteral lymph nodes. The DRE and PSA tests are uncomplicated and non-invasive and they should be done on all male patients over 50 with ureteral tumour to exclude the diagnosis of metastatic ureteral tumour from prostate cancer. There is limited value for TRUS to diagnose prostate cancer, however, it can be used when the DRE or PSA is abnormal.

\section{Conclusion}

We have reported an uncommon case of metastatic ureteral tumour caused by primary prostate cancer. The periureteral lymphatic pathway played an important role in the spread of prostate cancer to the proximal ureter. It is important to raise clinical awareness of the possibility of unusual metastatic sites in patients with primary prostate cancer.

Competing interests: Dr. Zhang declares no competing financial or personal interests.

This paper has been peer-reviewed.

\section{References}

1. Jemal A, Center MM, DeSantis C, et al. Global patterns of cancer incidence and mortality rates and trends. Cancer Epidemiol Biomarkers Prev 2010;19:1893-907. http://dx.doi.org/10.1158/10559965.EPI-10-0437

2. Sim HG, Cheng CWS. Changing demography of prostate cancer in Asia. Eur J Cancer 2005;41:834-45. http://dx.doi.org/10.1016/i.ejca.2004.12.033

3. Epstein JI. Pathology of prostatic neoplasia. In: Wein AJ, Kavoussi LR, Novick AC, Partin AW, Peters CA. Campbell-Walsh Urology. 9th ed. Philadelphia, PA: B. Saunders; 2007:2874-5.

4. DiSibio G, French SW. Metastatic patterns of cancers: Results from a large autopsy study. Arch Pathol Lab Med 2008;132:931-9.

5. Schneider S, Popp D, Denzinger $S$, et al. A rare location of metastasis from prostate cancer: Hydronephrosis associated with ureteral metastasis. Adv Urol 2012;2012:656023.

6. Stow B. Fibrolymphosarcomata of both ureters metastatic to a primary lymphosarcomata of the anterior mediastinum of thymus origin. Ann Surg 1909;50:901-6. http://dx.doi.org/10.1097/00000658190911000-00009

7. Hulse $C A, O^{\prime} N$ Neill TK. Adenocarcinoma of the prostate metastatic to the ureter with an associated ureteral stone. J Urol 1989;142:1312-3.

8. Cohen WM, Freed SZ, Hasson J. Metastatic cancer to the ureter: A review of the literature and case presentations. J Urol 1974;112:188-9.

9. Zollinger RW, Wise HA, Clausen KP. An unusual presentation of intrinsic ureteral obstruction secondary to carcinoma of the prostate: A case report. J Urol 1981;125:132-3.

10. Chalasani $V$, Macek $P, O^{\prime}$ Neill GF, et al. Ureteric stricture secondary to unusual extension of prostatic adenocarcinoma. Can J Urol 2010;17:5031-4.

Correspondence: Dr. Dexin Yu, Department of Urology, The Second Hospital of Anhui Medical University, China; yudx_urology@126.com 